\title{
Impact of nodal involvement on survival outcomes in chondrosarcoma: retrospective cohort analysis of Surveillance, Epidemiology, and End Results (SEER) database (2004-2015)
}

Lee D. Cranmer, MD, PhD, FACP ${ }^{a, b, \star}$, Bonny Chau, MPH ${ }^{a}$, Matthew J. Thompson, MDc Elizabeth T. Loggers, MD, PhD ${ }^{a, b}$, Seth M. Pollack, MD ${ }^{a, b}$, Michael J. Wagner, MDª,b Teresa S. Kim, MD ${ }^{d}$, Edward Y. Kim, MD,

Gabrielle M. Kane, MB, EdD, FRCPC ${ }^{\text {e }}$, Gabriel J. Pavey, MD ${ }^{c}$

\begin{abstract}
Objectives: Factors associated with nodal involvement in chondrosarcoma and outcomes based on treatment modality were analyzed using the Surveillance, Epidemiology, and End Results (SEER) database.

Methods: Chondrosarcomas involving axial and appendicular parts of the body diagnosed from 2004 to 2015 were identified in SEER. Clinical, pathologic, and treatment parameters were compared with respect to nodal status at initial diagnosis by Fisher's exact or Student's t-test. Disease-specific sunvival (DSS) and overall sunvival (OS) were evaluated by Kaplan-Meier analyses, and by Cox regression models. Results: Synchronous regional nodal metastases were present in $1.3 \%$ of chondrosarcoma patients. Lymph node involvement was associated with primary tumor location in extraskeletal tissue and the pelvis, and with distant metastasis and larger primary tumor size at diagnosis. Patients with nodal involvement had 5-year DSS of 48\% [95\% confidence interval (Cl): 28\%-65\%], versus 82\% (95\% Cl: 80\%-84\%) for those without (log-rank $P<0.001)$. 5-year OS with and without nodal involvement were 38\% (95\% Cl: 21\%-55\%) and 73\% (95\% Cl: $71 \%$ $75 \%)$, respectively (log-rank $P<0.001$ ). Surgical excision of nodes was associated with improved DSS and OS. Radiation and chemotherapy were not associated with improved DSS/OS.

Conclusions: The nodal disease is uncommon at presentation in chondrosarcoma. Greater clinical vigilance for regional nodal metastases may be warranted for those with specific risk factors, including extraskeletal or pelvic primary sites, myxoid, mesenchymal, or dedifferentiated histologies, and large size. Surgical excision of regional nodes is associated with improved DSS/OS. This analysis suggests a therapeutic effect of surgical treatment, rather than selection for favorable underlying biological factors.
\end{abstract}

Keywords: Chemotherapy, Radiotherapy, Adjuvant, Neoadjuvant, Surgery, Prognosis, Outcome, Survival, Cartilage, Myxoid, Mesenchymal

Chondrosarcoma is the second most common primary solid malignancy of bone, comprising $25 \%$ of all bone sarcomas ${ }^{[1]}$. The mainstay of management for patients with the localized disease

a Medical Oncology Division, University of Washington, ${ }^{b}$ Clinical Research Division, Fred Hutchinson Cancer Research Center, Departments of ${ }^{\circ}$ Orthopedics and Sports Medicine, 'S Surgery and ${ }^{e}$ Radiation Oncology, University of Washington, Seattle, WA

This report was presented in abstract and poster format at the Annual Meeting of the Connective Tissue Oncology Society in Rome, Italy November 14-17, 2018.

This manuscript has been peer reviewed.

Sponsorships or competing interests that may be relevant to content are disclosed at the end of this article.

${ }^{*}$ Corresponding author. Address: Medical Oncology Division, University of Washington, 825 Eastlake Ave. East, CE2-128, Seattle, WA 98109. Tel: 206-606-7439; fax: 206-606-6618. E-mail address: Icranmer@seattlecca.org (L.D. Cranmer).

Copyright $\odot 2020$ The Authors. Published by Wolters Kluwer Health, Inc. on behalf of IJS Publishing Group Ltd. This is an open-access article distributed under the terms of the Creative Commons Attribution-Non Commercial-No Derivatives License 4.0 (CCBY-NC-ND), where it is permissible to download and share the work provided it is properly cited. The work cannot be changed in any way or used commercially without permission from the journal.

International Journal of Surgery Oncology (2020) 5:e91

Received 23 March 2020; Accepted 2 June 2020

Published online 26 June 2020

Supplemental Digital Content is available for this article. Direct URL citations appear in the printed text and are provided in the HTML and PDF versions of this article on the journal's website, www.IJSOncology.com.

http://dx.doi.org/10.1097//J9.00000000000000091 remains surgery. For most tumors, wide excisions are preferred ${ }^{[2,3]}$. Low-grade chondrosarcomas of the extremities can be managed with more conservative surgical approaches, including intralesional excision with local adjuvant treatments ${ }^{[4-6]}$. Radiotherapy is used for patients with unresectable or symptomatic lesions, with most published studies focusing on chondrosarcoma of the spine and skull base ${ }^{[7,8]}$.

High-grade chondrosarcoma (grade III) is associated with a $32 \%-71 \%$ risk of regionally advanced or metastatic disease ${ }^{[9,10]}$. Chondrosarcomas, like other sarcomas, are generally viewed as having a hematologic route of dissemination, reflected by their propensity for pulmonary metastases. As a result, there is a paucity of data to guide the treatment of patients with uncommon clinical presentations, such as regional lymph node involvement at primary diagnosis.

Chondrosarcoma metastasis to regional lymph nodes is rare, with limited case reports reported in the literature (Fig. 1) ${ }^{[11-14]}$. There have been several reports of chondrosarcomas with lymph node involvement in nonhuman mammals ${ }^{[15-17]}$. The rarity of lymph node involvement from primary bone tumors may be attributed to the lack of lymphatic vessels in association with bones ${ }^{[18]}$. Nevertheless, Wan et al recently published an analysis of the Surveillance, Epidemiology, and End Results program (SEER) database, reporting that regional nodal involvement among chondrosarcoma cases occurred in $1.3 \%$ of cases, and was associated with inferior survival [hazard ratio (HR): $2.20,95 \%$ confidence interval (CI): $1.50-3.24]^{[19]}$. This study did not assess the impact of treatment 

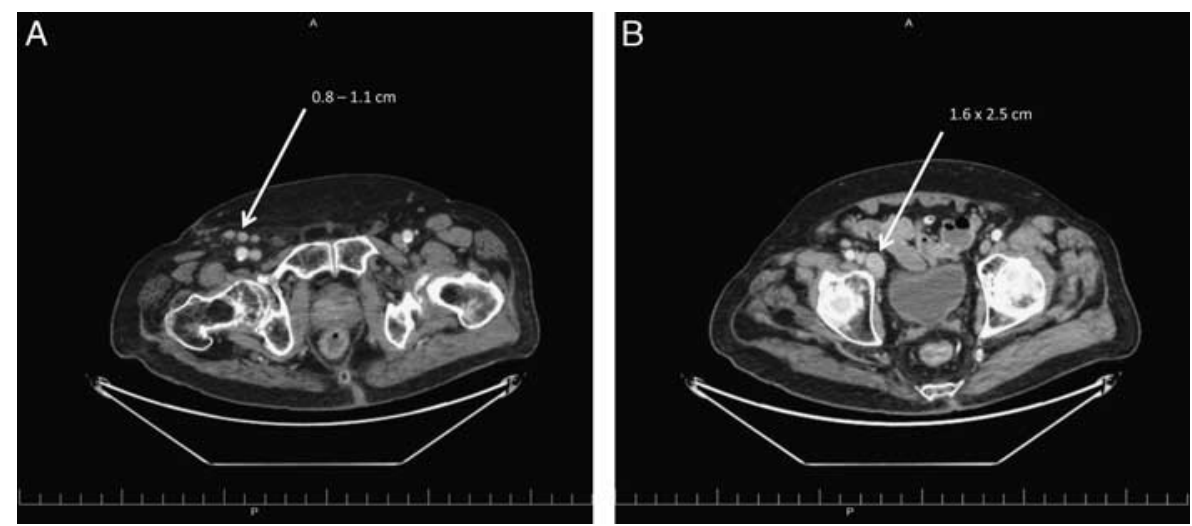

Figure 1. Computed tomographic images of pelvis. Male with right tibial dedifferentiated chondrosarcoma. Biopsy confirmed recurrence $\sim 4$ years after initial diagnosis. A, 3 right inguinal lymph nodes $(0.8-1.1 \mathrm{~cm}$ maximal dimension). B, Right external iliac lymph node $(1.6 \times 2.5 \mathrm{~cm}$ maximal dimension). Arrows indicate the locations of the index lesions within the respective image.

on outcome. In addition, the patients included in the study represented a 27-year timespan, from 1988 to 2015, in which treatment may have changed significantly.

This analysis sought to investigate this high-risk subpopulation of patients further, with specific attention toward the relationship between treatment modality and survival, focusing on those treated more recently. These findings will hopefully help guide management of this challenging subset of chondrosarcoma patients.

\section{Methods}

\section{Cohort}

For this retrospective cohort analysis, chondrosarcoma cases were extracted from the 1973 to 2016 SEER database. SEER is a publicly accessible database of de-identified data, for which human subjects review is not required. This research project has been registered with the Research Registry (http://www.researchregistry.com) and the unique identifying number is researchregistry 5162. This research adheres to the guidelines of the STROCSS Group in regard to the reporting of cohort studies in surgery ${ }^{[20]}$.

A total of 6526 chondrosarcoma cases were identified. Cases were excluded if diagnosed before 2004, due to our interest in patients diagnosed and treated more recently. Cases were also excluded from 2016, due to a change in variable coding in the SEER database, making direct comparisons with patient records from 2004 to 2015 unclear. In addition, cases were excluded if they had duplicate entries (identified using the SEER patient identification number), lacked information regarding survival, had tumor primary location in the head and neck (see Supplemental Materials Table 1 for list of primary tumor sites included, Supplemental Digital Content 1, http://links.lww.com/ IJSO/A13), or reported an unknown lymph node status. A total of 2399 unique cases diagnosed between the years 2004 and 2015 met inclusion criteria (Fig. 2).

\section{Variables}

Demographic and clinical characteristics evaluated in this study included: age at diagnosis, sex, race, year of diagnosis, tumor location, histologic type, distant metastasis, grade, and treatment modalities. Treatment modalities of interest were: surgery of the primary site, lymph node surgery, receipt of radiation, and receipt of chemotherapy. An orthopedic oncologic surgeon categorized primary tumor location, primary tissue origin, and primary bone site for our analysis, based on the primary tumor site listed in SEER. Tumor size was dichotomized according to the American Joint Committee on Cancer (AJCC) guidelines for bone cancer staging as being $<8.0 \mathrm{~cm}$ or $\geq 8.0 \mathrm{~cm}^{[21]}$.

Disease-specific survival (DSS) was the timespan from the time of diagnosis to death from chondrosarcoma. Patients dying from

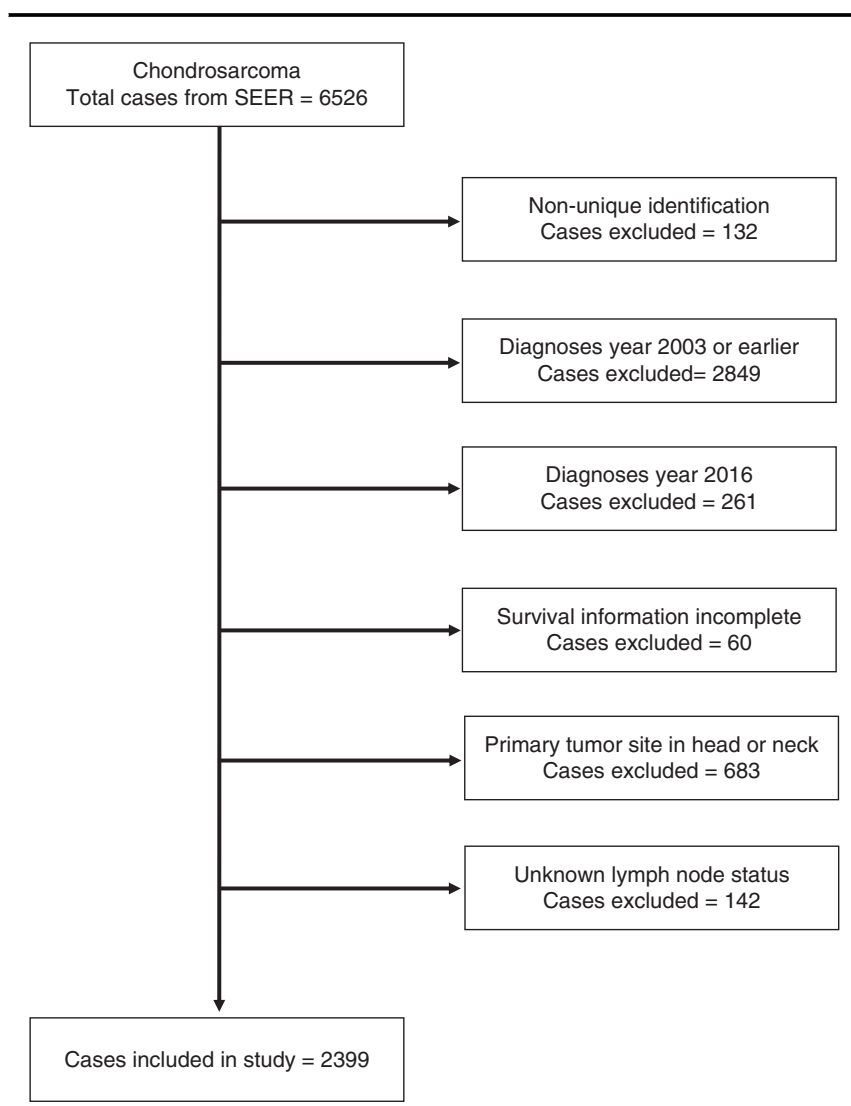

Figure 2. Selection process for inclusion of cases. SEER indicates Surveillance, Epidemiology, and End Results Program. 
Table 1

Comparison of demographic, clinical, and pathologic characteristic in patients with or without nodal involvement.

\begin{tabular}{|c|c|c|c|}
\hline Characteristic & $\begin{array}{c}\text { No Nodal } \\
\text { Involvement, } \\
\mathrm{N}=\mathbf{2 3 6 7}(\%)\end{array}$ & $\begin{array}{c}\text { Nodal } \\
\text { Involvement, } \\
\mathrm{N}=\mathbf{3 2}(\%) \\
\end{array}$ & $P$ \\
\hline \multicolumn{4}{|l|}{ Age at diagnosis } \\
\hline Mean \pm SD (age range) & $53 \pm 18(4-98)$ & $57 \pm 14(28-82)$ & 0.2688 \\
\hline \multicolumn{4}{|l|}{ Sex } \\
\hline Male & $1335(56)$ & $20(62.5)$ & 0.591 \\
\hline Female & $1032(44)$ & $12(37.5)$ & \\
\hline \multicolumn{4}{|l|}{ Race } \\
\hline Black & $183(8)$ & $2(6)$ & 0.925 \\
\hline White & 2031 (86) & 29 (91) & \\
\hline Other/unknown & $153(6)$ & $1(3)$ & \\
\hline \multicolumn{4}{|l|}{ Year of diagnosis } \\
\hline 2004-2009 & $1121(47)$ & $16(50)$ & 0.859 \\
\hline 2010-2015 & $1246(53)$ & $16(50)$ & \\
\hline \multicolumn{4}{|l|}{ Primary tumor location } \\
\hline Appendicular & $1423(60)$ & $17(53)$ & 0.044 \\
\hline Axial & $923(39)$ & $13(41)$ & \\
\hline Other/unknown & $21(1)$ & $2(6)$ & \\
\hline \multicolumn{4}{|l|}{ Primary tissue origin } \\
\hline Extraskeletal & $244(10)$ & $10(31)$ & 0.001 \\
\hline Skeletal & $2123(90)$ & $22(69)$ & \\
\hline \multicolumn{4}{|l|}{ Primary bone site* } \\
\hline Upper extremity & $437(21)$ & $3(14)$ & $<0.001$ \\
\hline Lower extremity & $736(35)$ & $4(18)$ & \\
\hline Ribs/sternum & $413(20)$ & $1(5)$ & \\
\hline Spine & $102(5)$ & 0 & \\
\hline Pelvis & $397(19)$ & $12(55)$ & \\
\hline Bone, NOS & $21(.1)$ & $2(9)$ & \\
\hline \multicolumn{4}{|l|}{ Histologic type } \\
\hline Chondrosarcoma & $1731(73)$ & $14(44)$ & 0.002 \\
\hline $\begin{array}{l}\text { Juxtacortical } \\
\text { chondrosarcoma }\end{array}$ & $30(1)$ & 0 & \\
\hline Chondroblastoma, malignant & $26(1)$ & 0 & \\
\hline Myxoid chondrosarcoma & $293(12)$ & $12(38)$ & \\
\hline $\begin{array}{l}\text { Mesenchymal } \\
\text { chondrosarcoma }\end{array}$ & $50(2)$ & $2(6)$ & \\
\hline Clear cell chondrosarcoma & $38(2)$ & 0 & \\
\hline $\begin{array}{l}\text { Dedifferentiated } \\
\text { chondrosarcoma }\end{array}$ & $199(8)$ & $4(13)$ & \\
\hline \multicolumn{4}{|l|}{ Distant metastasis } \\
\hline No & 2189 (92) & $19(59)$ & $<0.001$ \\
\hline Yes & $163(7)$ & $13(41)$ & \\
\hline Unknown & $15(1)$ & $0(0)$ & \\
\hline \multicolumn{4}{|l|}{ Grade } \\
\hline Grade I & $736(31)$ & $1(3)$ & $<0.001$ \\
\hline Grade ॥ & 817 (35) & $7(22)$ & \\
\hline Grade III & $261(11)$ & $8(25)$ & \\
\hline Grade IV & $192(8)$ & $5(16)$ & \\
\hline Unknown & $361(15)$ & $11(34)$ & \\
\hline \multicolumn{4}{|l|}{ Size } \\
\hline $0.0-8.0 \mathrm{~cm}$ & $1136(48)$ & $5(16)$ & $<0.001$ \\
\hline$\geq 8.0 \mathrm{~cm}$ & $873(37)$ & $25(78)$ & \\
\hline $\begin{array}{l}\text { No mass/tumor found/ } \\
\text { unknown }\end{array}$ & $358(15)$ & $2(6)$ & \\
\hline \multicolumn{4}{|l|}{ Lymph node surgery } \\
\hline No & 2257 (95) & $17(53)$ & $<0.001$ \\
\hline Yes & $110(5)$ & $15(47)$ & \\
\hline \multicolumn{4}{|l|}{ Surgery } \\
\hline No & $294(12)$ & $14(44)$ & $<0.001$ \\
\hline Yes & $2070(87)$ & $18(56)$ & \\
\hline Missing & $3(0)$ & $0(0)$ & \\
\hline
\end{tabular}

Table 1

(Continued)

\begin{tabular}{lccc}
\hline Characteristic & $\begin{array}{c}\text { No Nodal } \\
\text { Involvement, } \\
\mathbf{N}=\mathbf{2 3 6 7}(\%)\end{array}$ & $\begin{array}{c}\text { Nodal } \\
\text { Involvement, } \\
\mathbf{N = 3 2}(\%)\end{array}$ & $\boldsymbol{P}$ \\
\hline Radiation & & & \\
$\quad$ No/unknown & $2042(86)$ & $21(66)$ & 0.003 \\
$\quad$ Yes & $325(14)$ & $11(34)$ & \\
Chemotherapy & & & \\
$\quad$ No/unknown & $2158(91)$ & $22(69)$ & $<0.001$ \\
$\quad$ Yes & $209(9)$ & $10(31)$ & \\
\hline
\end{tabular}

*Excludes primary tumors not located on bones. No nodal involvement $n=2106$, nodal involvement $n=22$.

NOS indicates not otherwise specified.

other causes were censored at the time of death in analyses of DSS. Overall survival (OS) was the timespan from diagnosis to death from any cause. In both DSS and OS analyses, patients lost to follow-up were censored at the last follow-up date indicated in SEER.

\section{Statistical analyses}

Baseline characteristics of lymph node positive cases were compared with lymph node negative and lymph node unknown cases using Fisher's exact or Student's t-tests. A Bonferroni correction accounted for multiple comparisons. DSS and OS were estimated using univariable Cox proportional hazards models and evaluated by subpopulation with the Kaplan-Meier method and logrank test.

Multivariable Cox proportional hazards analyses were limited to 2 independent variables among those lymph node positive cases, due to the limited number of such cases and events for each outcome (DSS: 15 events/32 cases; OS 22 events/32 cases) ${ }^{[22]}$. The likelihood ratio (LR) test was used to compare the full and reduced Cox regression models. A value of $P \leq 0.05$ was designated for rejection of null hypotheses, with correction (ie, Bonferroni), if appropriate. Statistical analyses were conducted with Stata version 12.1 (StataCorp, College Station, TX).

\section{Results}

\section{Baseline characteristics}

Of the 2399 included cases, the majority were males with a mean age of 54 years and the median year of diagnosis in 2010. Thirtytwo $(1.3 \%)$ cases were lymph node positive $(\mathrm{LN}+)$, while 2367 cases were lymph node negative (LN - ) (Table 1). The appendicular parts of the body were the most common anatomic sites of the primary tumor $(60 \%$ of the total population; $53 \% \mathrm{LN}+$ and $60 \% \mathrm{LN}-$ ). Tumors originated primarily in skeleton irrespective of nodal status ( $89 \%$ of the total population).

\section{Factors associated with nodal involvement}

Several clinical and pathologic variables were disproportionately represented in the $32 \mathrm{LN}+$ patients: extraskeletal tumor location, presence of distant metastasis at diagnosis, higher tumor grade, and increased primary tumor size (Table 1). Among those with a skeletal primary site, $\mathrm{LN}+$ patients were most likely to have the pelvis as primary site $(12 / 22,55 \%)$. Myxoid, mesenchymal, and 
Table 2

Univariable Cox analyses of DSS and OS.

\begin{tabular}{|c|c|c|c|c|c|}
\hline & $\mathbf{n}$ & DSS HR (95\% Cl) & DSS 5-Year (95\% Cl) & OS HR (95\% Cl) & OS 5-Year $(95 \% \mathrm{Cl})$ \\
\hline \multicolumn{6}{|c|}{ Entire Study Population, $n=2399$} \\
\hline \multicolumn{6}{|c|}{ Lymph node status } \\
\hline No & 2367 & Referent & $82 \%(80 \%-84 \%)$ & Referent & $73 \%(71 \%-75 \%)$ \\
\hline Yes & 32 & $4.22(2.52-7.06)$ & $48 \%(28 \%-65 \%)$ & $3.74(2.44-5.72)$ & $38 \%(21 \%-55 \%)$ \\
\hline \multicolumn{6}{|c|}{ Lymph node positive population, $n=32$} \\
\hline \multicolumn{6}{|c|}{ Lymph node surgery } \\
\hline No & 17 & Referent & $19 \%(3 \%-45 \%)$ & Referent & $16 \%(3 \%-39 \%)$ \\
\hline Yes & 15 & $0.19(0.06-0.61)$ & $76 \%(42 \%-92 \%)$ & $0.42(0.17-1.00)$ & $60 \%(32 \%-80 \%)$ \\
\hline \multicolumn{6}{|l|}{ Surgery } \\
\hline No & 14 & Referent & $16 \%(1 \%-47 \%)$ & Referent & $13 \%(1 \%-40 \%)$ \\
\hline Yes & 18 & $0.16(0.05-0.50)$ & $68 \%(39 \%-85 \%)$ & $0.26(0.10-0.67)$ & $56 \%(31 \%-75 \%)$ \\
\hline \multicolumn{6}{|l|}{ Radiation } \\
\hline No/unknown & 21 & Referent & $49 \%(22 \%-71 \%)$ & Referent & $34 \%(14 \%-55 \%)$ \\
\hline Yes & 11 & $1.32(0.47-3.68)$ & $45 \%(17 \%-71 \%)$ & $0.67(0.27-1.67)$ & $45 \%(17 \%-71 \%)$ \\
\hline \multicolumn{6}{|l|}{ Chemotherapy } \\
\hline No/unknown & 22 & Referent & $45 \%(22 \%-66 \%)$ & Referent & $38 \%(18 \%-58 \%)$ \\
\hline Yes & 10 & $0.64(0.20-2.02)$ & $53 \%(17 \%-79 \%)$ & $0.65(0.25-1.68)$ & $40 \%(12 \%-67 \%)$ \\
\hline
\end{tabular}

$\mathrm{Cl}$ indicates confidence interval; DSS, disease-specific survival; HR, hazard ratio; OS, overall survival.

dedifferentiated histologic subtypes were more common among $\mathrm{LN}+$ versus $\mathrm{LN}$ - patients.

$\mathrm{LN}+$ patients were more likely to have received early multimodality treatment with lymph node surgery, radiation therapy, and chemotherapy as part of their primary treatment. In contrast, such patients were less likely to have received surgery of the primary site than $\mathrm{LN}$ - chondrosarcoma patients. Among those with lymph node involvement, 18 received any type of surgery, 15 received lymph node surgery, and 14 patients did not undergo any type of surgical treatment.

\section{Survival outcomes relative to nodal status}

The 5-year DSS and OS of all chondrosarcoma cases included were $82 \%$ (95\% CI: $80 \%-83 \%$ ) and $72 \%$ (95\% CI: 70\%-74\%), respectively. In univariable analyses, nodal involvement was associated with inferior DSS and OS (DSS HR: 4.22, 95\% CI:
2.52-7.06 and OS HR: 3.74, 95\% CI: 2.44-5.72, Table 2). Fiveyear DSS rate for $\mathrm{LN}+$ and $\mathrm{LN}$ - cases were $48 \%(95 \% \mathrm{CI}$ : $28 \%-65 \%$ ) and $82 \%$ (95\% CI: $80 \%-84 \%$ ), respectively (logrank $P<0.001$, Fig. 3A). The 5-year OS rates were 38\% (95\% CI: $21 \%-55 \%$ ) for $\mathrm{LN}+$ cases and $73 \%$ (95\% CI: $71 \%-75 \%)$ for those without nodal involvement (log-rank $P<0.001$, Fig. 3B).

\section{Survival outcomes in $L N+$ patients relative to treatment modality}

Receipt of lymph node surgery was associated with superior survival among LN + cases (DSS HR: 0.19, 95\% CI: 0.06-0.61and OS HR: 0.42, 95\% CI: 0.17-1.00) (Table 2; Fig. 4). The median DSS was 110 months (95\% CI: 44 mo-NA) for those who received lymph node surgery and 8 months (95\% CI: $2-29 \mathrm{mo}$ ) for cases that did not. Receipt of any surgery was also associated with superior DSS and OS
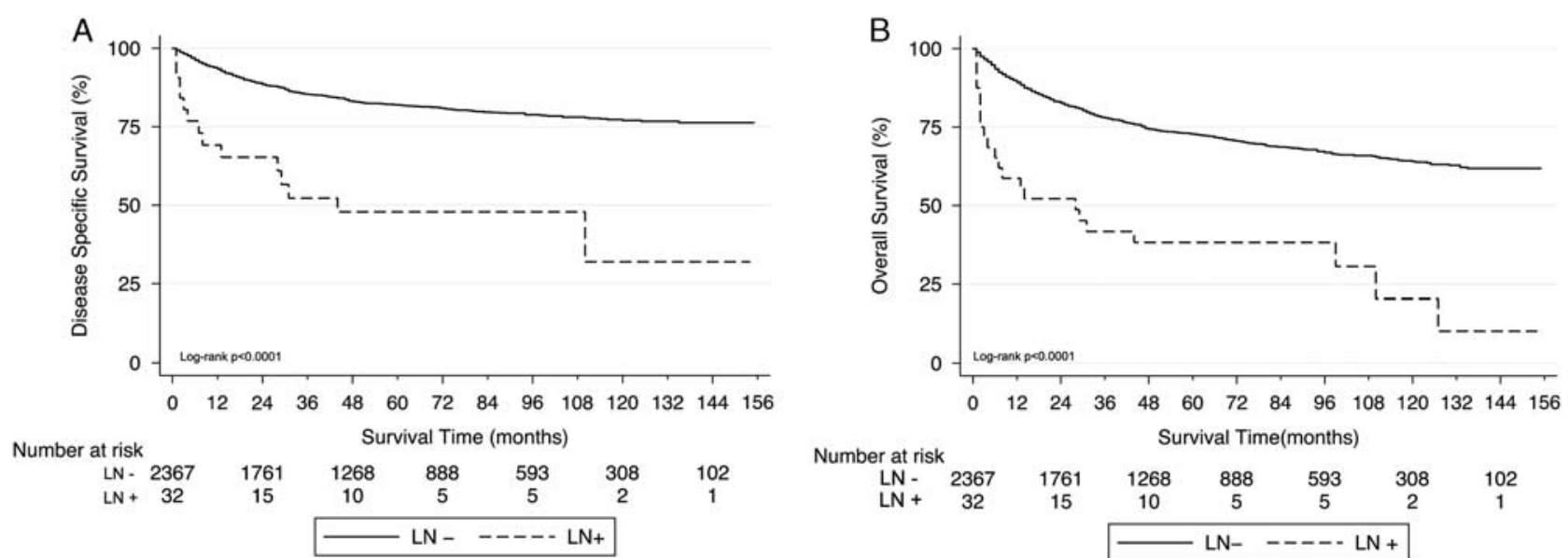

Figure 3. Survival outcomes by status of lymph nodes at initial diagnosis. A, Disease-specific survival. B, Overall survival. LN + indicates lymph node positive; LN - , lymph node negative. 

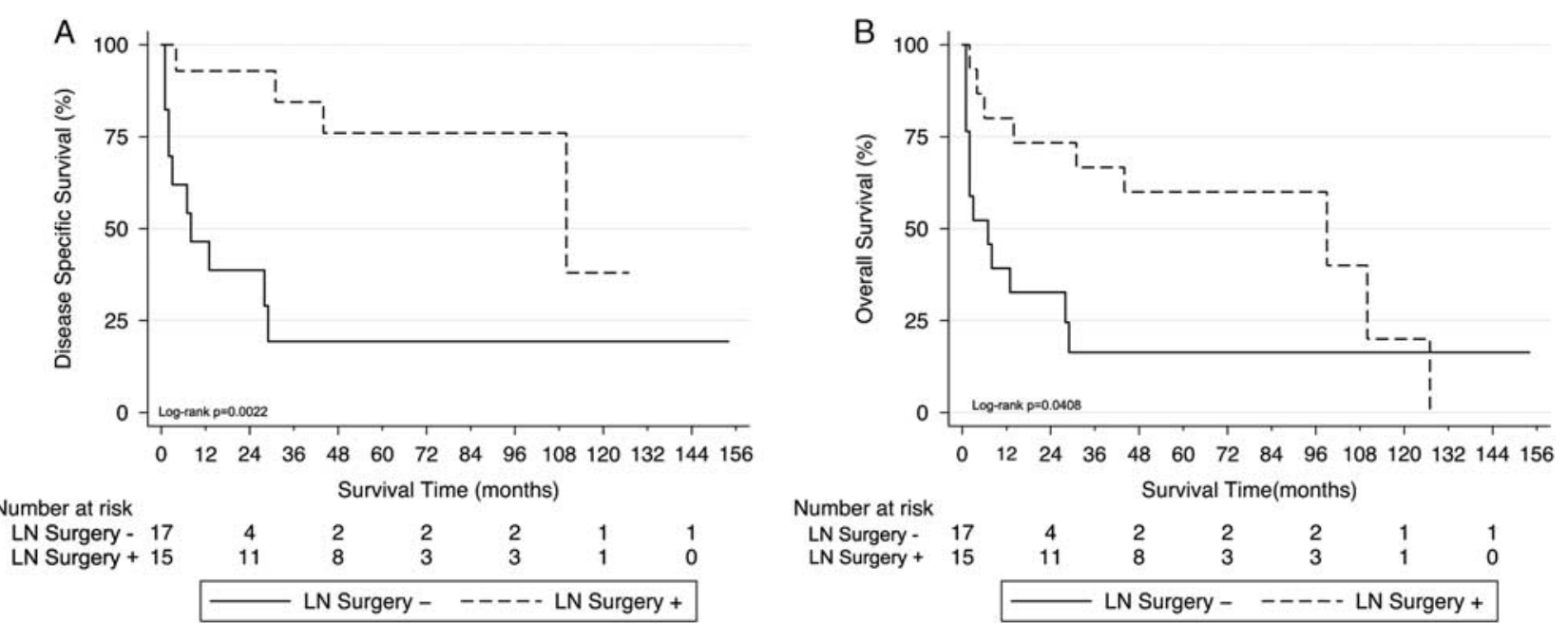

Figure 4. Survival outcomes by receipt of lymph node surgery in patients with lymph nodes involved by chondrosarcoma at initial diagnosis. A, Disease-specific survival. B, Overall survival. LN Surgery + indicates treated with lymph node surgery; LN Surgery -, not treated with lymph node surgery.

survival among those with positive nodal involvement (DSS HR: 0.16, 95\% CI: 0.05-0.50 and OS HR: 0.26, 95\% CI: 0.10-0.67, Table 2).

Patients with distant metastases at diagnosis were less likely to receive surgical treatment than those without distant metastases (Supplemental Materials Table 2, Supplemental Digital Content 1, http://links.lww.com/IJSO/A13). This was true both for the entire study population $(P<0.0001)$ and for the $\mathrm{LN}+$ cohort $(P=0.029)$. In $\mathrm{LN}+$ patients, lymph node surgery was less likely in those with distant metastases at diagnosis $(P=0.005$; Supplemental Materials Table 3, Supplemental Digital Content 1, http://links.lww.com/IJSO/A13). Among the entire study population, the likelihood of lymph node surgery was not associated with the presence of distant metastases $(P=0.375)$.

Multivariable Cox analyses were conducted to assess the impact of surgical treatment and the presence of distant metastases at diagnosis among $\mathrm{LN}+$ patients (Table 3 ). For both DSS and OS, receipt of surgery was associated with improved survival outcomes. In contrast, the presence of distant metastases was not significantly associated with DSS or OS. The LR test indicated that the inclusion of distant metastasis status in the model did not improve the model fit versus surgery status alone.

\section{Table 3}

Multivariable Cox analyses of DSS and OS.

\begin{tabular}{lcc}
\hline & DSS HR $\mathbf{( 9 5 \%} \mathbf{~ C l})$ & OS HR $\mathbf{( 9 5 \% ~ C l )}$ \\
\hline Surgery & & \\
$\quad$ No & Referent & Referent \\
Yes & $0.20(0.05-0.71)$ & $0.27(0.09-0.76)$ \\
Distant metastases & & \\
No & Referent & Referent \\
Yes & $1.59(0.49-5.14)$ & $1.01(0.38-2.68)$ \\
LR test vs. surgery alone & $P=0.43$ & $P=0.99$ \\
\hline
\end{tabular}

Lymph node positive population, $n=32$

*LR test with one degree of freedom.

$\mathrm{Cl}$ indicates confidence interval; DSS, disease-specific survival; HR, hazard ratio; LR, likelihood ratio; OS, overall survival.
Treatment with radiation therapy or chemotherapy was not associated with improved DSS or OS compared with those not receiving these modalities or with unknown treatment status. Receipt of chemotherapy and radiation therapy were not associated with one another among all patients with distant metastases at diagnosis $(\mathrm{n}=176, P=0.516$, Supplemental Materials Table 4A, Supplemental Digital Content 1, http://links.lww.com/ IJSO/A13) or among those $\mathrm{LN}+$ patients $(\mathrm{n}=32, P=0.703$, Supplemental Materials Table 4B, Supplemental Digital Content 1, http://links.lww.com/IJSO/A13). LN + patients receiving chemotherapy $(n=10)$ included those in 4 major histologic subclassifications (Supplementary Materials Table 5, Supplemental Digital Content 1, http://links.lww.com/IJSO/A13).

\section{Discussion}

We used the SEER database to explore lymph node involvement in the primary diagnosis of chondrosarcoma. As anticipated, regional lymph node involvement was uncommon, being evident in only $1.3 \%$ of cases in the SEER database. These findings are consistent with prior reports ${ }^{[19]}$. The subset of patients presenting with such involvement was distinct from those lacking lymph node involvement. Specifically, cases with lymph node involvement were more commonly extraskeletal, larger, higher grade, arising in the axial skeleton, of myxoid or dedifferentiated histologic subtypes, and more commonly metastatic at initial presentation.

Collectively, these data suggest that lymph node involvement is associated with more aggressive and more advanced disease at initial presentation. Consistent with this, the presence of lymph node involvement was associated with inferior DSS and OS (Fig. 3). Biologic variation in histologic type may be one explanatory factor, with myxoid, mesenchymal, and dedifferentiated chondrosarcomas representing a greater proportion of those with lymph node involvement. Indeed, myxoid chondrosarcomas have been known to behave differently than other types of chondrosarcomas, including conventional chondrosarcomas ${ }^{[23]}$. 
A key objective of this analysis was the identification of recommendations for treatment in $\mathrm{LN}+$ patients. Only primary tumor surgery and lymph node surgery were associated with improved outcomes among $\mathrm{LN}+$ patients. Surgery was more commonly undertaken in those without metastatic disease at presentation. The beneficial association of surgery seen in multivariable Cox analyses, after controlling for distant metastasis status, supports a protective effect of surgical treatment itself, rather than simply selecting for those with inherently better prognoses.

Receipt of lymph node surgery was inversely associated with distant metastasis status in those with lymph node involvement, again potentially indicating selection for lymph node surgery those with an inherently better prognosis. That improved survival outcomes were associated specifically with lymph node surgery is important, as such therapy is intended to treat directly the clinical scenario of interest herein, lymph node involvement. This beneficial effect was detectable despite receipt of lymph node surgery by less than half $(47 \%, 15 / 32)$ of $\mathrm{LN}+$ patients. Taken together, these data support an actual therapeutic effect of lymph node surgery in those chondrosarcoma patients with lymph node involvement. The limited sample size of $\mathrm{LN}+$ patients and limited selection of explanatory variables in SEER prevent a more detailed analysis of other potential confounders.

Neither radiation therapy nor chemotherapy was associated with improved DSS or OS. It was possible that radiation therapy and chemotherapy might be administered preferentially in conjunction to those with advanced disease. However, there was no association between the use of these 2 treatment modalities among all patients with distant metastases at presentation, or among $\mathrm{LN}+$ patients. It is therefore less likely that these treatments are being selectively administered to those with a poor prognosis.

The guidelines of the National Comprehensive Cancer Network (NCCN) recognize the potential utility of radiation therapy in the management of chondrosarcoma, especially those with limited surgical resectability ${ }^{[21]}$. Our results do not exclude benefit. Instead, our results suggest that its use be adapted to the clinical situation of a particular patient.

In contrast, NCCN guidelines state " $[t]$ here is no established chemotherapy regimens for conventional chondrosarcoma (grades 1-3)" (see page MS-8) ${ }^{[21]}$. Other chondrosarcoma subtypes reputedly display some sensitivity to chemotherapy, with mesenchymal chondrosarcoma more responsive to Ewing sarcoma regimens and dedifferentiated chondrosarcoma more responsive to osteosarcoma regimens ${ }^{[21,24,25]}$. Sirolimus and cyclophosphamide ${ }^{[26]}$ or dasatinib ${ }^{[27]}$ have modest activity in high-grade chondrosarcomas and are used clinically. Recently, a potential role for immunotherapy has been reported ${ }^{[28]}$. Again, while our analysis does not indicate benefit for the use of chemotherapy in chondrosarcoma patients generally, there may be specific clinical situations justifying such use. The histologic subtype may be one such criterion that clinicians use to select patients for chemotherapy treatment.

This analysis must be interpreted in light of its limitations. First, the number of $\mathrm{LN}+$ chondrosarcoma patients is small. As a result, we are unable to distinguish the independent effect of lymph node positivity in the absence of further metastatic disease. The limited sample size also prevented stratification by histologic subtypes. The subtypes included are recognized as having differential sensitivity to radiation and chemotherapy, as noted above. Therefore the statistical power to detect beneficial effects for radiation therapy or chemotherapy is further limited. That being said, surgical therapy was associated with improved outcomes, serving effectively as a positive control. If radiation therapy or chemotherapy have any beneficial effects when applied generally to $\mathrm{LN}+$ chondrosarcoma patients, the effect size is significantly less then that of surgical treatment.

Second, these anlyses are retrospective, reflecting cases diagnosed in 2004-2015. This time frame was selected because (1) treatment patterns and modalities during that period likely reflect current practice; and (2) changes in SEER classifications of cases and covariates outside this interval might introduce greater variability into the analyses. Changes in treatment patterns could affect the applicability of the results, but we are unaware of breakthrough insights that might alter the conclusions.

Another significant limitation of SEER is the granularity of treatment information available. For radiation therapy, information regarding the mode of treatment administrations is available, but the limited number of $\mathrm{LN}+$ chondrosarcoma cases did not allow us to consider different radiation treatment protocols. For chemotherapy, only receipt or nonreceipt/unknown are coded. Thus, despite the broad potential meaning of "radiation therapy" or "chemotherapy," these data do not allow more granular exploration of such treatments.

SEER classifies both radiation therapy and chemotherapy treatment as "yes" versus "no/unknown." Concerns have been raised regarding the investigation of radiation therapy and chemotherapy using data from SEER ${ }^{[29]}$. This relates to questions regarding misclassification, with a comparison between SEER and a Medicare claims database (the latter acting as a "gold standard") indicating up to $10 \%$ of cases are misclassified with respect to receipt of either radiation therapy or chemotherapy. This study was conducted in patients with cancer of the bladder, female breast, colorectal, lung, ovarian pancreatic, and prostate. There are several compelling reasons to undertake analyses such as this using SEER data.

First, primary regional lymph node involvement is an uncommon presentation of an uncommon type of disease (chondrosarcoma). Opportunities to explore clinical parameters, outcomes, and treatment are therefore limited. Even a large sarcoma treatment center is unlikely to see enough $\mathrm{LN}+$ chondrosarcoma patients to undertake meaningful investigations. Thus, these data allow such exploration, even with limitations.

Second, the concerns raised regarding the misclassification of SEER treatment data were raised in a study using insurance claims data as a gold standard comparator ${ }^{[29]}$. However, there exist reports of significant misclassification associated with insurance claims databases ${ }^{[30]}$. Thus, the comparator used as "gold standard" may not be as reliable as hoped. That $90 \%$ of cases in the study were classified correctly implies that significant information exists within the SEER radiation therapy and chemotherapy classifications.

In the current study, neither radiation nor chemotherapy was associated with improved survival. Lack of association may be explained by 3 potential reasons: there is actually no benefit; there is a benefit, but misclassification prevented its detection; or there is benefit from certain types of therapy in certain subsets of patients, with inadequate numbers of cases available to detect a modest treatment effect. The last 2 explanations are not mutually exclusive. A modest treatment effect might have existed, but either the total number of cases receiving particular treatments 
were inadequate or misclassification tended to minimize the differences between groups receiving or not receiving the particular treatment. Both effects would tend to lead to an underpowered assessment of these modalities. Both limitations could be overcome with a considerable increase in the number of patients receiving treatment for nodal involvement. On the basis of the rarity of this circumstance, such data are unlikely to become available.

In conclusion, lymph node involvement in patients with chondrosarcomas is uncommon, but does occur disproportionately in patients with large tumors, high grade, and nonconventional disease histologies, including myxoid, mesenchymal, and dedifferentiated. Synchronous distant metastases are more common in node-positive patients, likely reflecting more aggressive disease biology. The presence of regional node involvement in those with chondrosarcoma is associated with inferior DSS and OS versus those without such involvement.

It is the practice at our center to investigate for lymph node metastasis only when concerns for such lesions are raised by suggestive clinical or radiological evidence. Given the low frequency of lymph node involvement in chondrosarcoma, additional measures, such as sentinel lymph node biopsy, do not appear to be warranted. The utility of further measures to riskstratify patients for lymph node involvement would require additional investigation.

In those with nodal involvement at presentation, surgical treatment, including lymph node surgery, is associated with improved survival outcomes. The results of this analysis suggest this is an actual effect of surgical treatment, rather than selecting for those with inherently better prognosis. In contrast, no beneficial effects were detected from either radiation therapy or chemotherapy, although a small benefit cannot be excluded. In the uncommon circumstance of chondrosarcoma patients presenting with lymph node involvement, surgical intervention should be the mainstay of treatment. Radiation therapy and chemotherapy should be tailored to the unique needs of specific patients.

\section{Ethical approval}

All research described herein was undertaken using data from SEER (https://seer.cancer.gov). SEER is a publicly accessible database of de-identified data, for which human subjects review and ethical approval are not required.

\section{Source of funding}

No specific funding source was used in the conduct of this research project.

\section{Author contribution}

L.D.C., B.C., and G.J.P.: conception and design, data acquisition. L.D.C., B.C., M.J.T., E.T.L., S.M.P., M.J.W., T.S.K., E.Y.K., G.M.K., and G.J.P.: analysis and interpretation of data. L.D.C., B.C., M.J.T., E.T.L., S.M.P., M.J.W., T.S.K., E.Y.K., G.M.K., and G.J.P.: drafting, revision, and final approval of manuscript.

\section{Conflict of interest disclosure}

Dr L.D.C. receives research funding, paid to institution, from Eli Lilly, AADi, BluePrint Medicine, Iterion, Gradalis, Philogen, Advenchen Laboratories, and CBA Pharma. Dr L.D.C.'s institution has received funding from Eli Lilly for conduct of clinical trials. Dr L.D.C. has received honoraria or has served on advisory boards for BluePrint Medicines and Regeneron. Dr S.M.P. receives research funding from Merck, EMD Serono, Incyte, Presage, Janssen, Oncosec and Juno. He has received honoraria or has served on advisory boards for Eli Lilly, Seattle Genetics, Bayer, Tempus, Daiichi Sankyo and Blueprint Medicine. M.J.W. receives research funding, paid to institution, from Adaptimmune, Athenex, Athenex, Deciphera, BluePrint, Incyte, Glaxo SmithKline. $\mathrm{He}$ has received travel support from GSK and Adaptimmune. $\mathrm{He}$ has received honoraria or has served on advisory boards for Adaptimmune and Deciphera. The remaining authors declare that they have no financial conflict of interest with regard to the content of this report.

\section{Research registration unique identifying number (UIN)}

researchregistry5162 (http://www.researchregistry.com).

\section{Guarantor}

Not applicable.

\section{References}

[1] Fletcher CDM. World Health Organization, International Agency for Research on Cancer. WHO Classification of Tumours of Soft Tissue and Bone, 4th ed. Lyon: IARC Press; 2013.

[2] Fiorenza F, Abudu A, Grimer RJ, et al. Risk factors for survival and local control in chondrosarcoma of bone. J Bone Joint Surg Br 2002;84:93-99.

[3] Andreou D, Ruppin S, Fehlberg S, et al. Survival and prognostic factors in chondrosarcoma: results in 115 patients with long-term follow-up. Acta Orthop 2011;82:749-55.

[4] Veth R, Schreuder B, van Beem H, et al. Cryosurgery in aggressive, benign, and low-grade malignant bone tumours. Lancet Oncol 2005;6: 25-34.

[5] Hickey M, Farrokhyar F, Deheshi B, et al. A systematic review and metaanalysis of intralesional versus wide resection for intramedullary grade I chondrosarcoma of the extremities. Ann Surg Oncol 2011;18:1705-9.

[6] Chen X, Yu LJ, Peng HM, et al. Is intralesional resection suitable for central grade 1 chondrosarcoma: A systematic review and updated metaanalysis. Eur J Surg Oncol 2017;43:1718-26.

[7] Indelicato DJ, Rotondo RL, Begosh-Mayne D, et al. A prospective outcomes study of proton therapy for chordomas and chondrosarcomas of the spine. Int J Radiation Oncol Biol Phys 2016;95:297-303.

[8] Amichetti M, Amelio D, Cianchetti M, et al. A systematic review of proton therapy in the treatment of chondrosarcoma of the skull base. Neurosurg Rev 2010;33:155-65.

[9] Evans HL, Ayala AG, Romsdahl MM. Prognostic factors in chondrosarcoma of bone: a clinicopathologic analysis with emphasis on histologic grading. Cancer 1977;40:818-31.

[10] Angelini A, Guerra G, Mavrogenis AF, et al. Clinical outcome of central conventional chondrosarcoma. JSurg Oncol 2012;106:929-37.

[11] Gulia A, Puri A, Jain S, et al. Chondrosarcoma of the bone with nodal metastasis: the first case report with review of literature. Indian J Med Sci 2011;65:360-4.

[12] Matsumoto K, Hukuda S, Ishizawa M, et al. Parosteal (juxtacortical) chondrosarcoma of the humerus associated with regional lymph node metastasis. A case report. Clin Orthop Related Rese 1993;290:168-73. 
[13] Bhosale SJ, Kshirsagar AY, Sulhyan SR, et al. Metaplastic carcinoma with predominant chondrosarcoma of the right breast. Case Reports 2010;3: 277-81.

[14] Fuzesi L, Rixen H, Kirschner-Hermanns R. Cytogenetic findings in a metastasizing primary testicular chondrosarcoma. Am J Surg Pathol 1993;17:738-42.

[15] Meindel M, Pohlman L, DeBey B, et al. Regional nodal metastasis of humeral chondrosarcoma in a dog. J Am Anim Hosp Assoc 2015;51:346-51.

[16] Murakami T, Kobayashi Y, Chiba S, et al. Humeral chondrosarcoma in a Hokkaido brown bear (Ursus arctos yesoensis). J Vet Med Sci 2012;74: 1195-7.

[17] Schmid T, Hilbe M, Ohlert S, et al. Chondrosarcoma in the humerus of a goat. Vet Comp Orthop Traumatol 2010;23:273-6.

[18] Edwards JR, Williams K, Kindblom LG, et al. Lymphatics and bone. Hum Pathol 2008;39:49-55.

[19] Wan L, Tu C, Li S, et al. Regional lymph node involvement is associated with poorer survivorship in patients with chondrosarcoma: a SEER analysis. Clin Orthop Relat Res 2019;477:2508-18.

[20] Agha R, Abdall-Razak A, Crossley E, et al. STROCSS 2019 Guideline: strengthening the reporting of cohort studies in surgery. Int J Surg 2019;72:156-65.

[21] National Comprehensive Cancer Network (NCCN). NCCN Guidelines Version 1.2020 Staging Bone Cancer, 2020. Available at: www.nccn.org/ professionals/physician_gls/pdf/bone.pdf. Accessed June 1, 2020.
[22] Harrell FE Jr. Regression Modeling Strategies: with Applications to Linear Models, Logistic Regression, and Survival Analysis. New York, NY: Springer-Verlag; 2001.

[23] Weiss SW, Goldblum JR. Cartilaginous soft tissue tumors. In: Weiss SW, Goldblum JR, editors. Soft Tissue Tumors, 5th ed. Philadelphia, PA: Mosby Elsevier; 2008.

[24] Wagner MJ, Livingston JA, Patel SR, et al. Chemotherapy for bone sarcoma in adults. J Oncol Pract 2016;12:208-16.

[25] Italiano A, Mir O, Cioffi A, et al. Advanced chondrosarcomas: role of chemotherapy and survival. Ann Oncol 2013;24:2916-2.

[26] Bernstein-Molho R, Kollender Y, Issakov J, et al. Clinical activity of mTOR inhibition in combination with cyclophosphamide in the treatment of recurrent unresectable chondrosarcomas. Cancer Chemother Pharmacol 2012;70:855-60.

[27] Schuetze SM, Bolejack V, Choy E, et al. Phase 2 study of dasatinib in patients with alveolar soft part sarcoma, chondrosarcoma, chordoma, epithelioid sarcoma, or solitary fibrous tumor. Cancer 2017;123: 90-7.

[28] Wagner MJ, Ricciotti RW, Mantilla J, et al. Response to PD1 inhibition in conventional chondrosarcoma. J Immunother Cancer 2018;6:94-97.

[29] Noone AM, Lund JL, Mariotto A, et al. Comparison of SEER treatment data with Medicare claims. Med Care 2016;54:e55-e64.

[30] Funk MJ, Landi SN. Misclassification in administrative claims data: quantifying the impact on treatment effect estimates. Curr Epidemiol Rep 2014;1:175-85. 